\title{
Therapeutic Approach against 2019-nCoV by Inhibition of ACE-2 Receptor
}

\author{
Authors \\ Gajendra Kumar ${ }^{1 \oplus}$, Dharmendra Kumar², Netra Pal Singh ${ }^{3}$
}

\author{
Affiliations \\ 1 Department of Chemistry, Krishna College, Bijnor, Uttar \\ Pradesh, India \\ 2 Science Branch, Archeological Survey of India, Patna \\ Zone, Behar, India \\ 3 Department of Chemistry, Deen Dayal Upadhyaya \\ Gorakhpur University, Gorakhpur, Uttar Pradesh, India
}

Key words

antiviral drug, $2019 \mathrm{nCoV}$, curcumin, catechin,

1-piperoylpiperidine, ACE-2 inhibitor

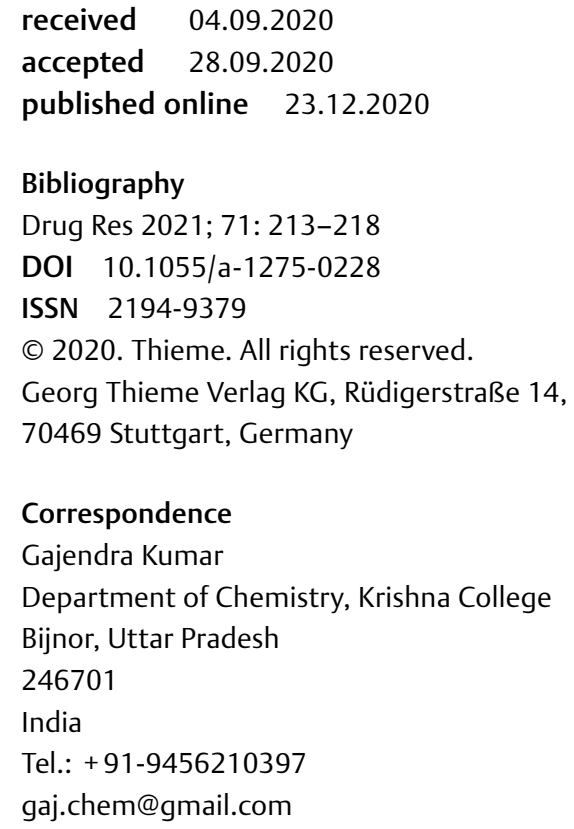

\begin{abstract}
The continued spread of 2019-nCoV has prompted widespread concern around the world. WHO formally named COVID-19 and International Committee on Taxonomy called it Severe Acute Respiratory Syndrome Coronavirus-2 (SARS-CoV-2). Due to this viral attack, the whole world is in lockdown. Presently, there is no effective way to control it, except social distancing and hygienic activity. World class scientists and researchers are trying to make vaccine and discover the medicine against the control and cure to this deadly viral disease. Our aim to presenting this article is kick-off deadly viral disease i.e COVID-19 by an easy way with minimum intervention and effort. Different ayurvedic therapeutic agents (Curcuma Longa L, Green tea and Piper nigrum) inhabit entry of virus in host cell, transmission of pathogen and improve the immunity. Curcumin and piperine (1-piperoylpiperidine) interact to each other and form a $\pi-\pi$ intermolecular complex which enhance the bioavailability of curcumin by inhibition of glucuronidation of curcumin in liver. Both the molecules curcumin and catechin get bound directly to receptors binding domain of S-protein and ACE-2 receptors of host cell, due to which these molecules inhibit the entry of virus in host cell i. e. animal survives from being infected.
\end{abstract}

\section{Introduction}

German veterinarians were puzzled in 1912, due to over the case of a feverish cat with extreme superfluous abdomen. That was the first time reported paradigm power of a coronavirus but veterinarians could not understand it at that time. It was few decades, coronavirus was giving chickens bronchitis and pigs an intestinal disease that killed almost every piglet under two weeks old and first time was identified animal coronavirus in 1930. Until 1960s, these pathogens remained an obscure link between animals and humans. In 1960, scientist's United Kingdom and the United States isolated two virus with corona like or crown like morphology were respon- sible for causing common colds in humans. The name coronavirus coined in 1968 is derived due to resemble the solar corona or crown like structure under electron microscope for the entire group of this virus. The sequence of the killer virus may be as follows: dog harms cats, the cat target pig intestines. The launching of severe acute respiratory syndrome (SARS) in 2003 caused by a new virus which belonged to coronavirus family had been playing role as a killer machine to kill the people and declared a pandemic, earlier believed that coronavirus causes only mild symptoms in humans [1]. Nowadays a new version of coronavirus family was produced, 


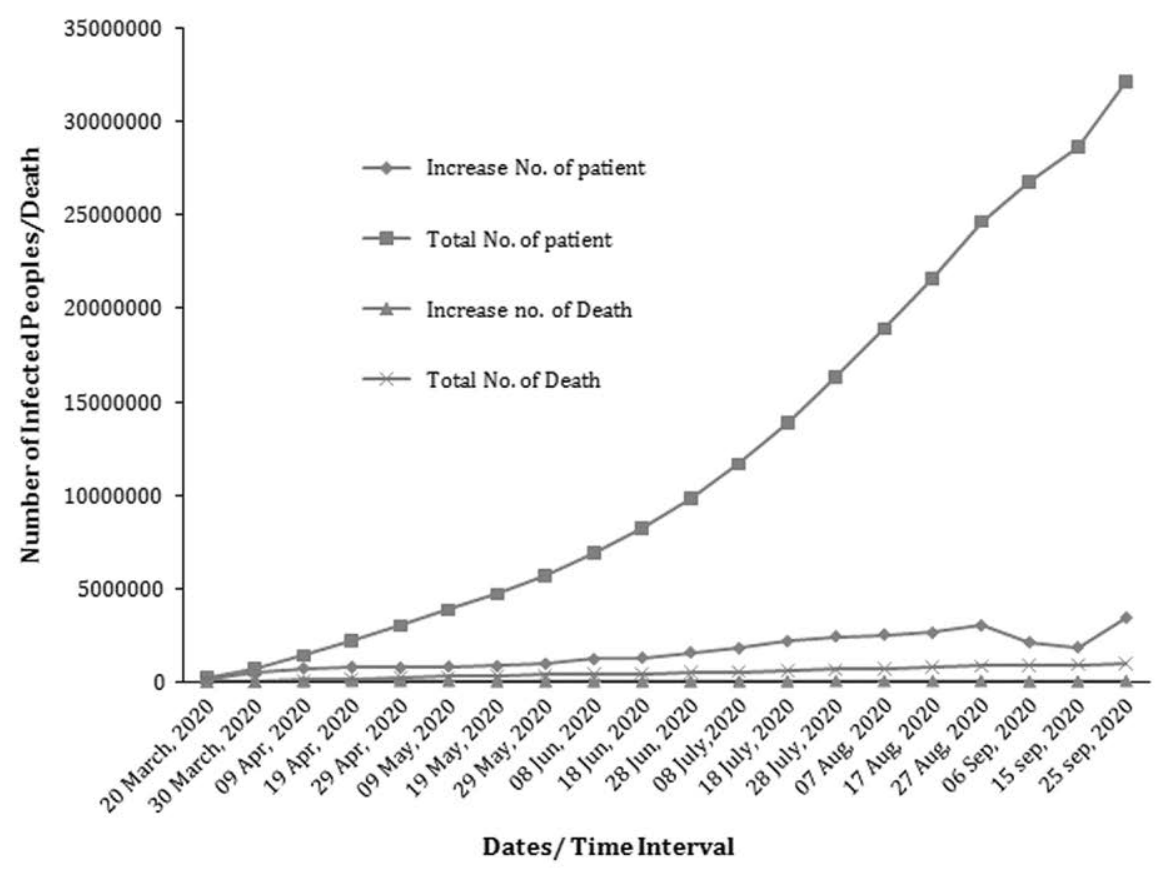

Fig. 1 Comparative study of total number of patients, total number of death, increase number of patients and increase number of death at different time interval.

a mutant may be by natural phenomenon or manmade virus with high spread power death toll from the COVID-19 pandemic. A 55 years old person from Hubei province could have been the first patient to have infected with the virus on November 17, 2019 the first case of novel severe acute respiratory syndrome emerged and then spread on the whole globe [2]. Doctor Jixian Zhang credited with first identifying the COVID-19 coronavirus has defended the country's response to the outbreak in an interview with state television on 27 December, amid international accusations that officials initially tried to conceal the crisis. After isolation and identification this pathogen originally called 2019 novel corona virus (2019nCoV) [3] but has subsequently been officially named severe acute respiratory syndrome coronavirus 2 (SARS-CoV-2) by the WHO. On $11^{\text {th }}$ February, 2020 the WHO announced a name for the new coronavirus disease called as COVID-19. On the same day, the coronavirus study group of the International Committee on Taxonomy of Viruses named 2019-nCoV as severe acute respiratory syndrome coronavirus 2 (SARS-CoV-2). As on February 2020 to 25 Sep. 2020 , 32110656 confirmed cases 980011 deaths have been reported for COVID-19 globally [4] (comparative study at different time interval reported in - Fig. 1). According to globally available data, COVID-19 caused by 2019-nCoV virus is the killer machine [5]. Possible ways to Inhibition of transmission: As per discussion above and urgency of outbreak has led to the empiric use of broad-spectrum antibiotics and antiviral agents some are clinically approved and some not approved in affected patients in several countries. There are four general approaches to cure and control this pandemic disease caused by the (SERS-COV-2) is as follows: 1 . Direct antiviral effects, 2 . Inhibition of viral entry and replication at the cellular level by targeting virus-related processes, 3 . Enhancement of host im- mune response and 4. Social distancing includes hygienic activities and complete lockdown [6]. At present time there are no antiviral agents of SARS-CoV-2 available. Scientists have been facing a big challenge to find possible treatments to save life and discover vaccines for future prevention of the virus, social distancing and complete lockdown is the only way to slow down the transmission of virus. Social distancing and lockdown is not an effective solution to kick off the virus and it is also not possible to lockdown for long time and not feasible for mankind \& economical aspects also. In this view, our aim was to introduce an easy and fast way to cure and control the action of aforesaid virus by triple action i. e. masking/ inhibition of Spike protein receptor binding domain (RBD) of Virus, ACE-2 receptor \& TMPRSS-2 Co-enzyme of host cell against to this pandemic disease using by ancient Ayurvedic remedy as soon as earlier along with theoretical scientific approach without any failure in the respect of inhibition of viral entry and replication at the cellular level by targeting virus-related processes along with enhance of host immunity. We are strongly recommended a therapy to cure and control the COVID-19, not for India but all over the globe in respect of mankind as well as our golden future.

\section{Ancient Ayurvedic Therapeutic Agents Curcuma Longa L. (Turmeric)}

Indian tradition believe that turmeric is a divine plant given to human being by God. Therefore, turmeric has always been a part of Vedic rituals in India. Sunlight dried powder of Curcuma longa $\mathrm{L}$ (Indian name haldi) is the good source of Curcumin [7]. Curcumin and its derivatives are found as effective inhibitor of triple target receptors to arrest the launch of SARs-COV-2, Angiotensin convert- 
ing enzyme-2 (ACE-2), TMPRSS2 receptors of host cells and Spike protein of virus. Curcumin and dimethoxy curcumin potently inhibits the activity of amino peptidase N (APN) /CD13. Curcumin has enough binding energy to each receptor and stronger inhibitory activities against COVID-19. So, it can be used as potential therapeutic agent against COVID-19 patient without adverse side effects.

\section{Black pepper (Piper Nigrum)}

Black pepper is used as medicine to treat digestive and respiratory tract related diseases caused by viral infection like as acute respiratory infection, asthma, chronic indigestion, and fever [8]. Black pepper is the good source of piperine (1-piperoylpiperidine), which is used as oral supplement to increase plasma level of coenzyme Q10. Black pepper Increase enzymatic activity, lipid per oxidation, antioxidant, bioavailability enhancer, immunomodulatory effect, WBC count and inhibit a dipogenesis [9]. Due to bioavailability effects of piperine it increases the level of curcumin and catechin in plasma and play a vital role to inhibit the entry of 2019-nCoV in cell.

\section{Green Tea}

Green tea contains a large number of compounds that is said to be beneficial to human health as Catechins, Caffeine, Theanine, Vitamins (Vitamin C, Vitamin $B_{2}$, Folic Acid, $\beta$-carotene, Vitamin E), Saponins, Fluorine, $\gamma$-amino butyric acid, Chlorophyll etc. Catechins are a type of polyphenol and the main astringency component in green tea, Catechin was first isolated from the Indian plant extract catechu. Catechin has duly binding affinity i. e. Spike protein RBD of Virus and ACE-2 Receptor of target cell. Catechin shows the potential inhibitory effect on COVID-19. Piperine component of Black Pepper has shown the bioavailability enhancer effect and increase the catechin concentration in tissues of the human body distribution with concomitant effect by inhibiting glucuronidation and gastrointestinal transit. Hence, both of them in combine form can be used as a potential drug against COVID-19 disease.

\section{Recipe to Treat COVID-19}

COVID-19 patient may be treated as following manner with ayurvedic recipe which will be shown the promising results along with the fast recovery. This recipe will also be beneficial for preventing the 2019-nCoV and to enhance the immune response.

Mixed $3 \mathrm{gm}$ fresh turmeric paste with $0.5 \mathrm{gm}$ fresh black pepper powder and equally divided it in to 3 part, each part of freshly prepared ayurvedic medicine introduce to patient orally with lukewarm cow milk thrice a day and $100 \mathrm{ml}$ green tea twice a day (boil $2 \mathrm{gm}$ dry leaf of green tea along with $0.25 \mathrm{gm}$ Black pepper in 100 $\mathrm{ml}$ water). After introducing this dose to the patient, take $2.5 \mathrm{gm}$ freshly prepared sun flower seed powder mixed it with lukewarm cow milk and introduce to the COVID-19 patient once a day. This aforesaid therapy will be promising results in respect of COVID-19 patient.

\section{Mechanism of the Pathogen}

Coronaviruses (CoVs) are relatively large viruses containing a single-stranded positive-sense RNA genome encapsulated within a nucleocapside protein and glycoprotein membrane envelope. The coronavirus genomes encodes four types of structural proteins nucleocapsid protein, membrane glycoprotein, envelope protein and spike protein in which spike protein is a major functional inducer of host immune responses [10]. Severe Acute Respiratory Syndrome caused by coronavirus is covered by enveloped spike glycoproteins (that give crown-like appearance) [11] essential for viral entry into the host cell that contains a variable receptor-binding domain (RBD) [6]. The RBD of Coronavirus binds to angiotensin converting enzyme 2 (ACE-2) receptor [12] and aminopeptidase $N$ (APN) another known name is CD13, dipeptidyl peptidase 4 (DPP4) $[13,14]$, carcinoembryonic antigen related cell adhesion molecule 1 (CEACAM1), Cellular serine protease found in the heart, lungs, kidney and gastrointestinal tract thus facilitating viral entry into the host cells $[15,16]$. The coronaviruses are also enveloped positive strand RNA virus with the largest genomes of RNA viruses characterized by 3-4 envelop binding and subsequent membrane fusion process of coronavirus are mediated by the spike membrane glycoprotein (Spike protein). The $S$ protein membrane contains three segments: a large ectodomain, a single pass transmembrane anchor and a short intracellular tail [15]. The ectodomain is distinct functional receptor-binding domain near the sub-unit Amino (S1 or $\mathrm{N}$ ) and a membrane fusion sub-unit carboxy (S2 or C) terminal [17]. These spike function to define viral tropism by their receptor specificity S1 or N terminal binds on the host cell surface by ACE-2 receptor for viral attachment and perhaps also by their membrane fusion by $\mathrm{S} 2$ or $\mathrm{C}$ terminal subunit activity virus genomes to enter into the host cell by the complex proteolytically processed by type2 transmembrane serine protease enzyme encoded by TMPRSS- 2 gene leading to cleavage of ACE-2 and activation of the Spike protein. Viral entry and cell infection trigger the host's immune system and the inflammatory cascade is initiated by antigen-presenting cells (APC). The process starts with the ACP performing two functions: first is presenting the foreign antigen to CD4 + T-helper cells and second one is releasing interleukin- 12 to further stimulate the T-helper cells. The T-helper cells stimulate CD8 + T-killer cells that will target any cell containing the foreign antigens. In addition, activated $\mathrm{T}$ helper cells stimulate beta cells to produce antigen-specific antibodies [18-20].

\section{Concomitant Effects of Curcumin \& Catechin with Piperine}

Curcumin has the poor absorption bioavailability due to rapid metabolism in liver and small intestinal wall. The major component of the black pepper is the alkaloid Piperine (1-pieroylpiperidine) enhance the bioavailability of the drugs by inhibition of glucuronidation in the liver and small intestine. Concomitant administration of turmeric powder with Piperine \& Catechin with Piperine increase the absorption bioavailability $2000 \%$ with no adverse side effects [21]. Due to increase the bioavailability, it will play indefinable promising role to cure and control the COVID-19 outbreak globally along with enhance the immune response.

\section{Molecular Bonding}

Ligand (curcumin or catechin) molecule linked to the site of ACE2 receptor which provides the way to virus entry in host cell. Similar- 


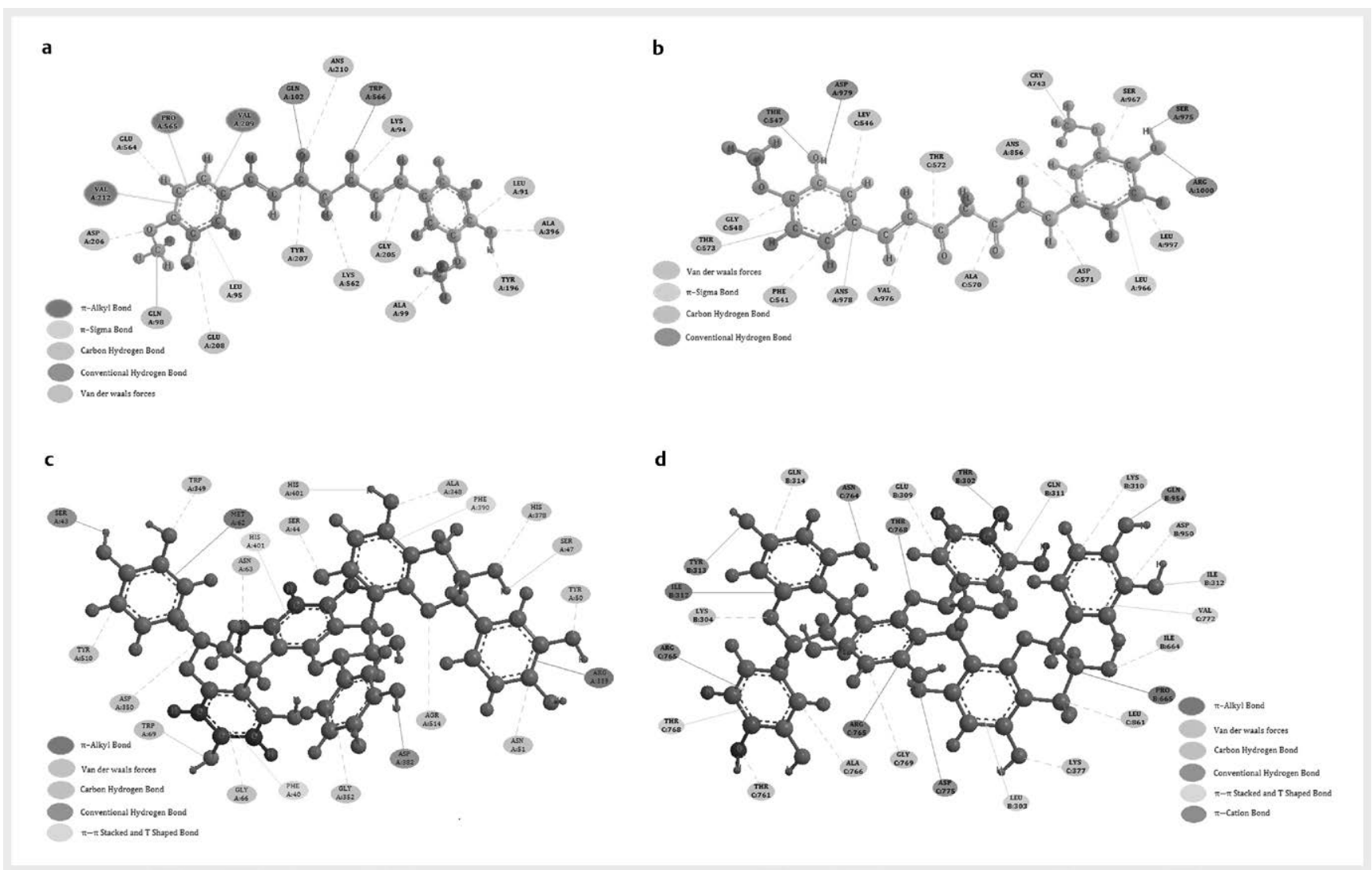

Fig. 2 Binding representation, a binding of ACE2 with Curcumin, $\mathbf{b}$ binding of S-protein with Curcumin, $\mathbf{c}$ binding of ACE2 with Catechin, $\mathbf{d}$ binding of S-protein with Catechin.

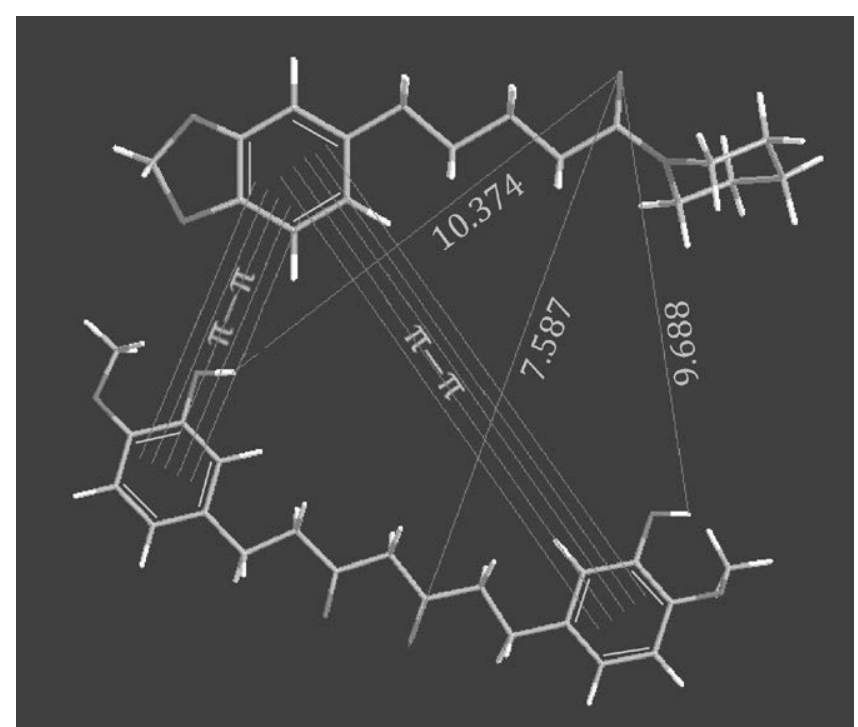

- Fig. 3 Intermolecular Complex formed by Curcumin and Piperine: The intermolecular distance between carbonyl group of Piperine to Enol group, two phenolic hydroxyl groups of Curcumin is $7.587 \AA$, 9.688 $\AA$ and $10.374 \AA$, respectively. ly it was also seen that ligand molecule binds to the RDB animo acid (S protein) which involved in host cell binding. Carbon hydrogen bond, conventional hydrogen bond and Van der waals force are formed between Curcumin or Catechin (ligand) and S-protein (amino acid). It also proves that ligands have strong bonding affinity with ACE-2 as well as S-protein but binding affinity of catechin is more than curcumin. Hence curcumin and catechin create inhibition environment between RBD and ACE- 2 receptors which cause the virus to not enter in the host cell ( $\triangleright$ Fig. 2).

The linkage of ACE-2 receptor and S-protein with ligand includes conventional $\mathrm{H}$-bond, Carban hydrogen bond, $\pi-\sigma$ bond, $\pi-a l k y l$ bond and $\pi-\pi$ interaction. Curcumin directly bonded to ACE- 2 receptor of host cell amino acids: ANS A:210, LYS A:94, LEU A:91, ALA A:396, LYS A:562, ALA A:99, TYR A:196, GLY A:205, TYR A:207, GLU A:208, GLU A:564, ASP A:206 (By Van der waals forces); GLN A:102, TRP A:566 (by conventional); GLN A:98 (C-H bond); VAL A:209, PRO A:565, VAL A:212 ( $\pi-a l k y l$ bond); LEU A:95 ( $\pi-\sigma$ bond). Catechin bonded to ACE-2 receptor of host cell amino acids: TRP A:349, SER A:44, ASP A:350, GLY A:352, HIS A:378, ALA A:348, AGR A:514, TYR A:510, TYR A:50, ANS A:51, ANS A:63 (By Van der waals forces); SER A:43, ASP A:382 (by conventional H-bond); HIS A:401, TRP A:69, SER A:47 (C-H bond); MET A:62, ARG A:393 (ח-Alkyl bond); HIS A:401, PHE A:390, PHE A:40 ( $\Pi-\pi$ stacket linkage); HIS A:401,

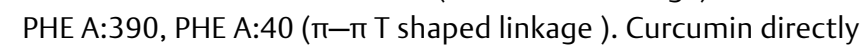
linked with S-protein of 2019-nCoV amino acids: LEU C:546, GLY C: 548, PHE C: 541, ANS A:856, LEU A:997, SER A:967, ASP C:571, 
ALA C:570, VAL A:976, THR C:572 ( by Van der Waals forces); ASP A:979, THR C:547, AGR A:1000, SER A:975 (by conventional H-bond); THR C:573, ASN A:978, CYS A:743 (by carbon-hydrogen bond); LER A:966 (by ח-sigma bond). Catechin linked to S-protein amino acids: GLN B:314, GLU B:309, LYS B:310, GLY B:311, ILE B:664, LYS C:733, LEU C:861, ASP B:950, GLY C:769, ALA C:766, LYS B:304, THR C:761 ( by Van der waals forces); TYR B:313, THR C:768, ASN C:764, THR B:302, GLN B:954, ASP C:775 (by conventional H-bond); ILE B:312 (by C-H bond); THR C:768, VAL C:772, LEU B:303 ( $\pi-\sigma$ bond); ARG C:765 ( $\pi-$ cation); ILE B:312, PRO B:665, ARG C:765 ( $\pi-$ alkyl bond). Hydroxyl group of catechin has high binding affinity to words the amini acid AGR-634 and VAL-635 of 2019-nCoV Spike protein. In addition, these two polyphenols are strong immune boosters and have been reported accounted for to initiate autophagy, another significant component of viral freedom [22-24]. Along these lines, it is evident that transmission of viral disease can be stop by using Curcuma Longa L, Green tea and Piper nigrum in daily life.

\section{Intercalating of Piperine with Curcumin}

So as to survey the intercalating affinity of piperine with curcumin as a transporter of the curcumin particle by way of metabolism, we have considered the idea of the intermolecular complex. A low binding energy $-3.1 \mathrm{kcal} / \mathrm{mol}$ complex is formed by $\pi-\pi$ linkage between the benzene ring of curcumin and piperine ( $\triangleright$ Fig. 3). As we find the distance between the enolic proton of curcumin to piperine to be shorter than the phenolic proton of curcumin and piperine, we recommend that the essential method of intercalation is through enolic intermolecular hydrogen holding and the sandwich $\pi-\pi$ linkage between the benzene rings of the curcumin and piperine molecules [25]. On account of this these collaborations among piperine and curcumin compete for intercalating between adjoining layers of curcumin thus providing for a channel for piperine to bind to curcumin in supporting its transportation through metabolic pathways. When glucuronosylation of curcumin is hindered by piperine, curcumin stays longer time in the body and provide more time for the absorption of curcumin thus increase the bioavailability of curcumin by 20 -flod through this method.

\section{Conclusion}

COVID-19 pandemic has caused a severe blow to social life, health and economic status. The malignancy of the virus can be detected only by the fact that it has killed many millions of people so far. Hence, there is a need to curb COVID-19 comprehensively. Our research suggest that the use of Curcuma Longa L (Curcumin), Piper Nigrum (Piperin) and Green Tea (Catechin) in their daily lives regularly can cure and prevent COVID-19 outbreak and infection.

\section{Acknowledgements}

No acknowledgments were reported by the authors.

\section{Conflicts of Interest}

The authors declare that they have no conflict of interest associated with this work.

\section{References}

[1] Cyranoski David. Profile of a scientists are quickly piecing together how the new coronavirus operates, where it came from and what it might do next - but pressing questions remain. Nature 2020; 581: 22-26

[2] Liying D, Shashu $\mathrm{H}$, Jianjun G. Discovering drugs to treat coronavirus disease 2019 (COVID-19). Drug Discoveries \& Therapeutics 2020; 14: $58-60$

[3] Zhou P et al. A pneumonia outbreak associated with a new coronavirus of probable bat origin. Nature 2020; https://doi.org/10.1038/ s41586-020-2012-7

[4] Rolling updates on corona virus disease (COVID-19) https://www.who. int/emergencies/diseases/novel-coronavirus-019/events-as-theyhappen

[5] Pengfei S, Xiaosheng L, Chao X et al. Understanding of COVID-19 based on current evidence. Jou Med Virol. 2020; 10.1002/jmv.25722.

[6] Shulla A, Sargent TH, Subramanya G et al. Transmembrane serine protease is linked to the severe acute respiratory syndrome coronavirus receptor and activates virus entry. J of Virology 2011; 85: 873-882

[7] Shiv K, Roop K, Mohammad M et al. Turmeric (Curcuma longa L.): A promising spice for phytochemical and pharmacological activitie. International Journal of Green Pharmacy 2013; 7: 85

[8] Priya NC, Kumar PS. Antiviral activities and cytotoxicaty assay of seed extracts of Piper longum and Piper nigrum on human cell lines. Int J Pharm Sci Rev Res 2017; 44: 197-202

[9] Joshi DR, Shrestha AC, Adhikari N. A Review on diversified use of the king of spices: Piper Nigrum (Black Paper). IJPSR 2018; 9: 4089-4101

[10] Liu Cynthia, Qiongqiong Z, Yingzhu L et al. Research and Development on Therapeutic Agents and Vaccines for COVID-19 and Related Human Coronavirus Diseases. ACS Cent Sci 2020; 6: 315-331

[11] Zheng YY, Ma YT, Zhang JY et al. COVID-19 and the cardiovascular system. https://doi.org/10.1038/s41569-020-0360-5

[12] Firas A, Mazhar S, Al Z et al. SARS-CoV-2 and Coronavirus Disease 2019: What We Know So Far. Pathogens. 2020; doi:10.3390/ pathogens 9030231

[13] Joong SS, Jin HK, Hyun YC et al. Irreversible Inhibition of CD13/ Aminopeptidase $\mathrm{N}$ by the Antiangiogenic Agent Curcumin. Chemistry \& Biology 2003; 10: 695-704

[14] Reguera J. Structural bases of coronavirus attachment to host aminopeptidase $\mathrm{N}$ and its inhibition by neutralizing antibodies. PLoSPathog 2012; doi: 10.1371/journal.ppat. 1002859

[15] Li Fang. Structure, Function, and Evolution of CoronavirusSpike Proteins. Annu Rev Virol 2016; 3: 237-261

[16] Aleksi S, Kvetoslava V, Petr B et al. Quaternary Benzo[c]phenanthridine Alkaloidsas Inhibitors of Aminopeptidase $\mathrm{N}$ andDipeptidyl Peptidase IV. Phytother Res 2002; 16: 84-87

[17] Thomas MG, Michal JB. Coronavirus spike proteins in viral entry and pathogenesis. Virology 2002; 279: 371-374

[18] Lingshu W et al. Evaluation candidate vaccine approaches for MERS-CoV. Nature. Communications 2015; 6: 7712. DOI: 10.1038/ ncomms 8712

[19] Kenneth EB, Zakir K], Duo YC et al. Angiotensin-converting enzyme in innate and adaptive immunity". Nat Rev Nephrol 2018; 14: 325-336 
[20] Emily LC, Eng EO, Chin YL et al. Inhibition of SARS coronavirus infection in vitro with clinically approved antiviral drugs. Emerging Infectious Diseases 2004; 10: 581-586

[21] Shobal G, david J, Thangam J et al. Influence of piperine on the pharmacokinetics of curcuminin animals and human volunteers. Planta Medica 1998; 64: 353-356

[22] Atala B], Namrata K, Vinayak $\mathrm{N}$ et al. Catechin and Curcumin interact with corona (2019-nCoV/SARS-CoV2) viral S protein and ACE2 of human cell membrane: Insights from Computational study and implication for intervention. 2020; https://doi.org/10.21203/ rs.3.rs-22057/v1
[23] Song JM. Anti-infective potential of catechins and their derivatives against viral hepatitis. ClinExp Vaccine Res 2018; 7: 37-42

[24] Shinojima N, Yokoyama T, Kondo Y et al. Roles of the Akt/mTOR/ p70S6K and ERK1/2 signaling pathways in curcumin induced. Autophagy, Autophogy 2007; 3: 635-637

[25] Vaishali MP, Sukanya D, Krishnan B. Quantum chemical and docking insights into bioavailability enhancement of curcumin by piperine in pepper. J of Phy Chem A 2016; 120: 3643-3653 